\title{
Dimensiones del autoritarismo, centralidad de la religión y orientaciones religiosas: diferencias en el análisis lineal y no lineal de sus relaciones
}

\author{
Authoritarianism dimensions, religion centrality \\ and religious orientation: Differences in linear and \\ nonlinear analysis of their relationships
}

\author{
Edgardo Etchezahar ${ }^{1}$ \\ Universidad de Buenos Aires - CONICET, Argentina
}

\author{
Silvina Brussino ${ }^{2}$ \\ Universidad Nacional de Cordoba - CONICET, Argentina
}

Resumen. La centralidad de la religión y las orientaciones religiosas han sido estudiadas en sus relaciones con el autoritarismo debido a que dichas expresiones de la religiosidad ofrecen un marco social convencional estable, el cual incluye una serie de pautas que rigen el comportamiento del sujeto autoritario. Sin embargo, estudios previos que analizaron estas relaciones no tuvieron en cuenta el aporte específico de cada una de las dimensiones del autoritarismo. El objetivo principal del estudio fue analizar las relaciones entre las dimensiones del autoritarismo, la centralidad de la religión y las orientaciones religiosas. Participaron 431 estudiantes universitarios, con edades comprendidas entre los 20 y 35 años, de los cuales el 72,38\% eran mujeres. Se observó que las dos dimensiones del autoritarismo se relacionan con la centralidad de la religión, la orientación religiosa intrínseca y extrínseca personal. Se discuten las relaciones entre las dimensiones, así como su análisis lineal y no lineal.

Palabras clave. Autoritarismo, orientaciones religiosas, centralidad de la religión, convencionalismo, agresión y sumisión autoritaria

Abstract. The centrality of religion and the religious orientations have been studied in its relations to authoritarianism because such expressions of religiosity offer a stable conventional social framework. Such framework includes a set of guidelines that govern the behavior of authoritarian subjects. However, previous studies examining these relationships did not take into account the specific contribution of each of the dimensions of authoritarianism. The main objective of the study was to analyze the relationship between the dimensions of authoritarianism, the centrality of religion and religious orientations. 431 college students participated, aged between 20 and 35 years, $72.38 \%$ of them were women. It was observed that the two dimensions of authoritarianism are related to centrality of religion, intrinsic and extrinsic religious orientation. Relationships between dimensions are discussed as well as their linear and nonlinear analysis.

Keywords. Authoritarianism, Religious orientation, Religion centrality, Conventionalism, Authoritarian aggression and submission

\footnotetext{
${ }^{1}$ Dr. Edgardo Etchehazar. . Universidad de Buenos Aires - CONICET. Direccion postal: Gral. Juan Lavalle, 2353, Buenos Aires. E-mail: edgardoetchezahar@psi.uba.ar

${ }^{2}$ Dr. Silvina Brussino. Laboratorio Psicología Cognitiva. Universidad Nacional de Córdoba - CONICET. E-mail: brussino@psyche.unc.edu.ar
} 


\section{Introducción}

El estudio psicológico de las relaciones entre el autoritarismo y diferentes expresiones de la religiosidad da inicio a principios del siglo XX y continúa hasta la actualidad (Dezutter, Robertson, Luyckx \& Hutsebaut, 2010). La razón principal para el análisis de las relaciones entre tales constructos radica en que los individuos autoritarios necesitan formar parte de marcos sociales convencionales estables que rijan al comportamiento. Según Duckitt (2010), este aspecto no implica que necesariamente los sujetos con mayores niveles de autoritarismo sean religiosos ni viceversa, ya que estudios que analizan las relaciones entre los constructos dan cuenta del amplio margen de religiosos que obtienen muy bajos puntajes en evaluaciones del autoritarismo y viceversa $(r=.38 ; p<.001)$ (Mavor, Macleod, Boal \& Louis, 2009).

Específicamente, desde una perspectiva psicológica, el autoritarismo se define por la presencia en un sujeto de tres conglomerados actitudinales: sumisión autoritaria, agresión autoritaria y convencionalismo. Tanto la sumisión autoritaria (tendencia a someterse a autoridades percibidas como legítimas), como la agresión autoritaria (hostilidad hacia las personas y grupos considerados como potenciales amenazas), han sido estudiadas como dos aspectos constitutivos de la dinámica autoritaria (Feldman, 2003), mientras que el convencionalismo (marco social de creencias compartidas) constituye el núcleo de creencias sobre las que se desarrolla dicha dinámica (Altemeyer, 2006; Etchezahar \& Brussino, 2013).

De las diferentes expresiones de la religiosidad, dos han sido estudiadas sistemáticamente en sus relaciones con el autoritarismo: la centralidad de la religión (Huber \& Huber, 2012) y las orientaciones religiosas (Maltby, 2002).

Por una parte, la centralidad de la religión en la vida del individuo refiere al nivel de integración del sistema de significados religiosos en la vida cotidiana de un sujeto (Huber, 2003; Huber \& Huber, 2012). De acuerdo con Park (2007), la centralidad de la religión delimita en qué medida un credo religioso constituye la visión del mundo de un sujeto. De esta manera, más allá de los diferentes credos religiosos (católico, judío, musulmán, entre otros) y sus respectivos manifiestos (Biblia, Torá, Corán, entre otros), la centralidad de la religión da cuenta la medida en que el sujeto los aplica a sus propias experiencias de vida. En este sentido, Altemeyer y Hunsberger (2005) consideran que una fuerte centralidad de la religión en la vida de un individuo es proclive al fundamentalismo religioso, ya que ambos se caracterizan por el rechazo a creencias que son consideradas diferentes a las que propone el credo al cual pertenecen.

Las relaciones entre el autoritarismo evaluado a través de la escala Autoritarismo del ala de derechas (RWA por sus siglas en inglés) y la centralidad de la religión, informadas en estudios previos, han sido entre moderadas (Johnson et al., 2011; $r=.33 ; p<.01$ ) $\mathrm{y}$ altas $(r=.76 ; p<.01$; Terrizzi, Shook \& Ventis, 2010), dependiendo del contexto donde se llevó a cabo cada investigación (Blogowska \& Saroglou, 2011; Coursey, 2013). Sin embargo, las evaluaciones del autoritarismo se realizaron con una versión de la escala RWA unidimensional (Altemeyer, 1981, 2006) que evalúa los tres conglomerados que componen el constructo (agresión, sumisión y convencionalismo) de manera simultánea, imposibilitando discriminar las relaciones de cada uno de ellos con la centralidad de la religión (Mavor et al., 2009).

Por otra parte, la orientación religiosa refiere al proceso que controla y organiza el comportamiento de aquellos individuos que adhieren a una religión a partir de sus creencias, diferenciando la orientación extrínseca e intrínseca (Allport \& Ross, 1967; Maltby \& Lewis, 1996). Según Maltby (2002) la Orientación Extrínseca (OE) caracteriza a los sujetos que consideran sus prácticas religiosas de manera instrumental, para alcanzar fines personales o sociales (por ejemplo, la aceptación grupal), mientras que la Orientación Intrínseca (OI) describe a aquellos individuos que interpretan la religión como un fin en sí mismo ( rezar en privado). A la vez, la OE se divide en dos factores de segundo orden asociados: la Orientación Extrínseca Personal (OEP) y la Orientación Extrínseca Social 
(OES). Mientras que el factor OEP remite al uso de la religión como seguridad, protección, comodidad o alivio, el factor OES da cuenta del uso de la religión como un medio social. Particularmente, una de las principales justificaciones para la distinción de las orientaciones religiosas ha sido el análisis de sus relaciones con el autoritarismo (Allport \& Ross, 1967; Mavor et al., 2009).

Estudios previos indican que las personas con mayor OI tienden a presentar mayores niveles de autoritarismo que aquellos sujetos con OE (Altemeyer, 1981; NúñezAlarcón, Moreno-Jiménez \& Moral-Toranzo, 2011; Sibley \& Duckit, 2008; Watson et al., 2003). Según Altemeyer (1996), esto sucede debido a que los niveles de sumisión autoritaria son elevados en los sujetos con OI, rechazando la idea de que las costumbres sociales puedan ser arbitrarias o que las de un grupo específico puedan ser tan buenas como las de otro, lo cual implica menores niveles de tolerancia y aceptación de la diferencia (Altemeyer \& Hunsberger, 2005).

Si bien ambas orientaciones pueden ser susceptibles de múltiples interpretaciones y prácticas autoritarias como no autoritarias, Abu-Raiya y Pargament (2011) consideran que la OI ofrece un marco privado, normativo y tradicional que no se observa en el caso de los sujetos con OE y que, en ocasiones, puede resultar un contexto que contribuya al desarrollo de ciertas tendencias autoritarias (Altemeyer, 1981; Mavor, Louis, \& Laythe, 2011). Sin embargo, al igual que con la centralidad de la religión, no se han desarrollado estudios que analicen las relaciones entre los constructos utilizando una versión de la escala RWA que discrimine las diferentes dimensiones del constructo para saber el aporte de cada una de ellas en la relación (Etchezahar, 2012).

Investigaciones actuales sobre la evaluación del autoritarismo (Etchezahar \& Brussino, 2013; Marasca, Marasca, \& Imhoff, 2013) permiten discriminar dos dimensiones del constructo (Agresión y Sumisión autoritaria por un lado y Convencionalismo por otro). Asimismo, se sugiere analizar las dimensiones del autoritarismo con otros constructos psicosociales de manera no lineal, por ejemplo estableciendo niveles bajos, medios y altos para cada dimensión del constructo (i.e. Agresión/Sumisión autoritaria y Convencionalismo), ya que en estudios previos se ha demostrado que este tipo de análisis permite avanzar en la comprensión del fenómeno autoritario en comparación a los análisis de tipo lineal comúnmente utilizados en el área de estudios (Mavor et al., 2011).

De acuerdo a lo expuesto, surge la interrogante sobre si las dimensiones del autoritarismo se relacionan con la centralidad de la religión y las orientaciones religiosas tanto de manera lineal como no lineal. Como hipótesis de trabajo, se propone que las relaciones entre las dimensiones del autoritarismo y las diferentes expresiones de la religiosidad difieren si se analizan de manera lineal y no lineal.

\section{Método}

\section{Participantes}

La muestra estuvo compuesta por 431 estudiantes universitarios, con edades comprendidas entre los 20 y 35 años $(M=22.88 ; D E=4.15)$, de los cuales el $72.38 \%$ eran mujeres $(n=312)$ y el $27.62 \%$ hombres $(n=119)$, residentes en la Ciudad Autónoma de Buenos Aires. Además, el 11.36\% $(n=49)$ se autoposicionó como perteneciente a clase social media-baja, el $81.43 \%(n=$ 351) a clase media y el $7.19 \%(n=31)$ a clase media-alta.

\section{Instrumentos}

Los datos fueron recolectados a través de un instrumento de evaluación de índole autoadministrable de lápiz y papel, asegurando el anonimato de los participantes. El mismo incluyó las siguientes variables:

Escala de autoritarismo del ala de derechas (RWA). Para evaluar el constructo se utilizó una versión reducida de la escala RWA (Altemeyer, 2002) compuesta por 14 ítems que evalúan dos dimensiones del constructo (Agresión/ Sumisión autoritaria y Convencionalismo), adaptada y validada al contexto local (Etchezahar, 2012). Para su validación se trabajó con dos muestras de estudiantes universitarios de la Ciudad de Buenos Aires $(N=880)$, cuya consistencia interna $(.75<\alpha<.81)$ y validez de constructo $(.97<$ CFI $<.98 ; .03<$ RMSEA $<.05)$ demostraron ser adecuadas. El formato de respuesta 
de la misma es tipo Likert con cinco anclajes de respuesta desde $1=$ "Totalmente en desacuerdo" a $5=$ "Totalmente de acuerdo". Puntuaciones mayores indican mayores niveles de autoritarismo en ambas dimensiones.

Escala de Orientación Religiosa (I-E 12). Se utilizó una versión de la escala Age Universal I-E-12 (Maltby, 2002) adaptada y validada para el contexto local (Simkin \& Etchezahar, 2013). La misma está compuesta por 12 ítems, seis de los cuales evalúan la Orientación Intrínseca (OI) (por ejemplo, "Mi religión es importante porque me da respuestas a muchas preguntas sobre el sentido de la vida", "Me esfuerzo por vivir mi vida acorde a mis creencias religiosas"), tres la Orientación Extrínseca Personal (OEP) (como, "Lo que la religión me ofrece principalmente es alivio en tiempos problemáticos y de tristeza", "Rezar es para obtener paz y felicidad") y tres la Orientación Extrínseca Social (OES) (por ejemplo, "Voy a la iglesia principalmente porque disfruto viendo a las personas que conozco ahí", "Voy a la iglesia principalmente para pasar tiempo con mis amigos").

Se utilizó como formato de respuesta el sugerido por Gorsuch y Venable (1983): tipo Likert con cinco opciones de respuesta, que van desde 1: Totalmente de acuerdo a 5: Totalmente en desacuerdo. Los resultados de la adaptación argentina de la escala (Simkin \& Etchezahar, 2013) indicaron propiedades psicométricas adecuadas en cuanto a su validez (S-B $\chi_{(\mathrm{gl})}^{2}=89.76_{(51)} ; \mathrm{CFI}=.97$; NNFI $=.97$; RMSEA $=$ $.057)$ y consistencia interna de sus dimensiones $(.79<$ $\alpha<$.88). La varianza total explicada de la escala total fue del $70.3 \%$, distribuyéndose en un $28.8 \%$ para el primer factor (OI), 22.7\% para el segundo (OEP) y $18.8 \%$ para el tercero (OES).

Centralidad de la religión (CR). Se incluyó la siguiente pregunta extraída de estudios realizados por el CIS (Centro Investigaciones Sociológicas, España): “En su opinión, ¿qué papel juega la religión en su vida?”. El formato de respuesta presenta un continuo de $1=$ "No me interesa la religión" a 5= "Ocupa un lugar clave en mi vida".
Variables socio-demográficas. Se desarrolló un cuestionario ad-hoc para indagar el sexo, la edad y la clase social autopercibida de los participantes.

\section{Procedimiento}

Los estudiantes universitarios que formaron parte de esta investigación participaron de manera voluntaria y anónima luego de brindar su consentimiento informado, pudiéndose retirar en cualquier momento. La administración de los instrumentos se realizó en un único encuentro. Asimismo, se les informó que los datos relevados serían utilizados con fines exclusivamente académico-científicos, bajo la Ley Nacional 25.326 de protección de datos personales.

Con la finalidad de cumplimentar los objetivos propuestos en este trabajo, se realizaron análisis líneas entre las variables de estudio (a través del estadístico $r$ de Pearson), así como análisis de tipo no lineal (a través del ANOVA de un factor con contraste posthoc Tukey b).

\section{Resultados}

A continuación la Tabla 1 presenta las relaciones entre las dimensiones del autoritarismo del ala de derechas, la centralidad de la religión y las orientaciones religiosas en los participantes (Tabla 1).

Las dos dimensiones de RWA se relacionan de forma positiva con la centralidad de la religión y con las orientaciones religiosas, indicando que a mayores niveles de Agresión/Sumisión autoritaria y Convencionalismo, mayor Centralidad de la Religión en la vida de los participantes, así como mayores niveles en las Orientaciones Religiosas (a excepción de la Orientación Extrínseca Social con la cual presenta una correlación baja), al realizar un análisis lineal entre los constructos.

Luego de conocer las relaciones entre las dimensiones del autoritarismo y las Orientaciones Religiosas de forma lineal (a través del estadístico $r$ de Pearson), se procedió a analizar si existían diferencias entre los niveles de las dimensiones (alto, medio y bajo) (Etchezahar, 2012) con cada orientación, a partir de un análisis no lineal (a través del $A N O V A$ de un factor). Para tal fin, en primer lugar se observó la distribución de las puntuaciones de la Orientación Intrínseca y los 
Tabla 1

Correlaciones entre las dimensiones de la escala $\mathrm{RWA}$, I-E 12 y la $\mathrm{CR}$

\begin{tabular}{lcccccc}
\hline & $\alpha$ & 1 & 2 & 3 & 4 & 5 \\
\hline 1. RWA Convencionalismo & .79 & - & & & & \\
2. RWA Agresión/Sumisión & .88 & $.32^{* *}$ & - & & & \\
3. CR & - & $.40^{* *}$ & $.31^{* *}$ & - & & \\
4. OI & .87 & $.43^{* *}$ & $.36^{* *}$ & $.75^{* *}$ & - & \\
5. OES & .72 & $.16^{*}$ & $.14^{*}$ & .05 & $.32^{* *}$ & - \\
6. OEP & .74 & $.29^{* *}$ & $.34^{* *}$ & $.58^{* *}$ & $.71^{* *}$ & $.22^{* *}$ \\
\hline
\end{tabular}

*. $p<.05 ; * * . p<.01$.

Nota. CR: Centralidad de la Religión; OI: Orientación Intrínseca; OES: Orientación Extrínseca Social; OEP: Orientación Extrínseca Personal.

grupos (bajo, medio y alto) conformados por ambas dimensiones del autoritarismo (Figura 1).

Como puede observarse en la Figura 1, la distribución de la OI difiere entre la dimensión Agresión/Sumisión y el Convencionalismo. Con la finalidad de analizar si estas diferencias son estadísticamente significativas se calcularon dos ANOVA de una vía.

Por una parte, la dimensión Agresión-Sumisión diferencia grupos con la Orientación Intrínseca $\left(F_{(2,}\right.$
229) $12.40 ; p<.001)$. A través de un contraste posthoc Tukey $b$, la OI se divide en dos grupos de acuerdo a las medias de la dimensión: niveles bajos $(M=21.17)$ y medios $(M=22.53)$, diferenciándose de quienes obtuvieron niveles altos $(M=27.29)$. Por otra parte, se observó que la dimensión Convencionalismo diferencia grupos con la Orientación Intrínseca $\left(F_{(2,22)}=21.14\right.$; $p<.001)$. A través de un contraste posthoc Tukey $b$, las puntuaciones en la Orientación Intrínseca se dividen en dos grupos de acuerdo a las medias de la dimensión
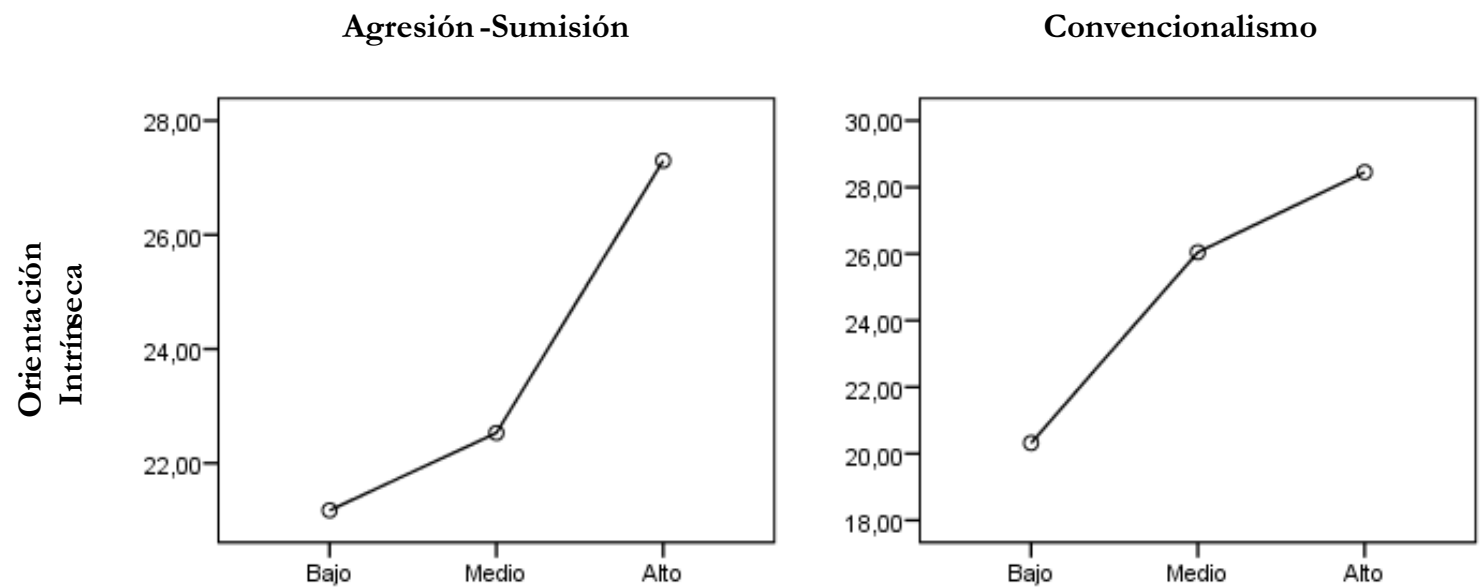

Figura 1. Orientación Intrínseca según niveles de Agresión/Sumisión autoritaria y Convencionalismo. Los valores extremos de la OI son 6 y 30 . 
Convencionalismo, conformando el primer grupo quienes obtuvieron puntajes medios $(M=26.01)$ y altos $(M=28.03)$, mientras que el segundo estuvo compuesto por quienes obtuvieron niveles bajos ( $M$ $=20.16)$.

En segundo lugar se analizó la distribución de la Orientación Extrínseca Social según los niveles de ambas dimensiones del autoritarismo (Figura 2).

\section{Agresión-Sumisión}

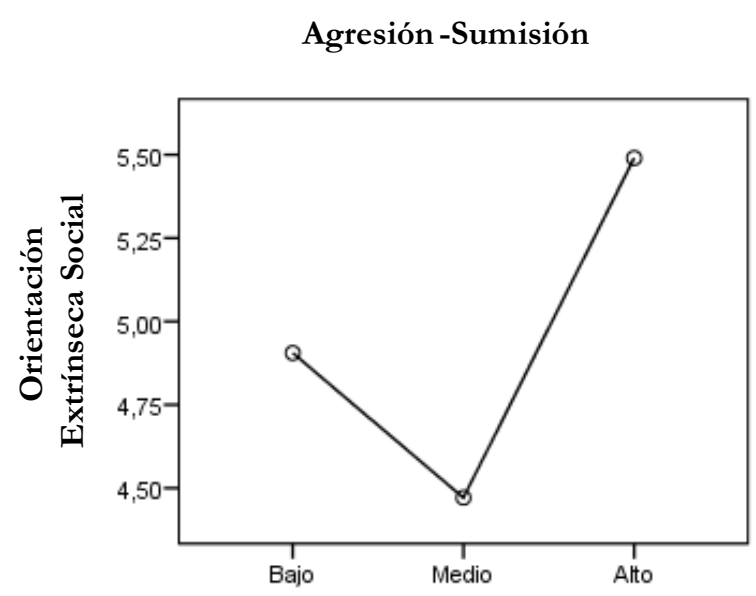

La distribución de la OES pareciera diferenciarse en la dimensión Agresión/Sumisión autoritariay Convencionalismo, sin embargo no se observaron diferencias estadísticamente significativas entre la OES y la primera $\left(F_{(2,243)}=1.33 ; p=\right.$ .264) o la segunda dimensión $\left(F_{(2,243)}=2.87 ; p=.058\right)$.

En tercer lugar, se estudió la distribución de la Orientación Extrínseca Personal y los niveles de las dimensiones del autoritarismo (Figura 3).

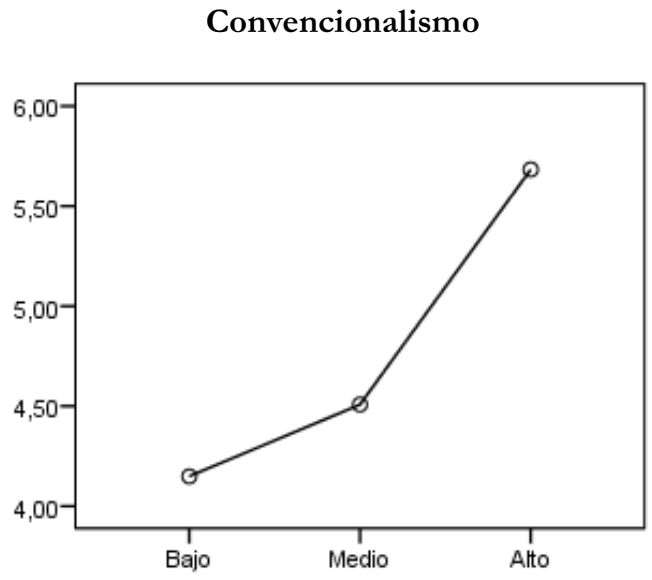

Figura 2. Orientación Extrínseca Social según niveles de Agresión/Sumisión autoritaria y Convencionalismo. Los valores extremos de la OES son 3 y 15.

Agresión-Sumisión

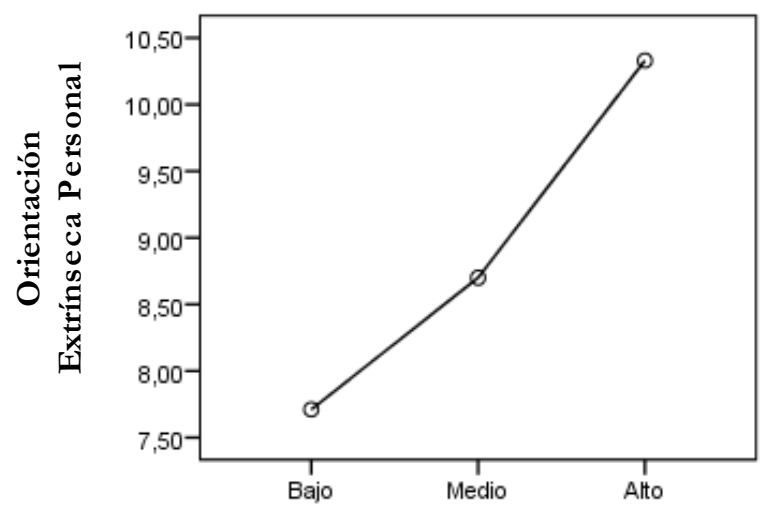

Convencionalismo

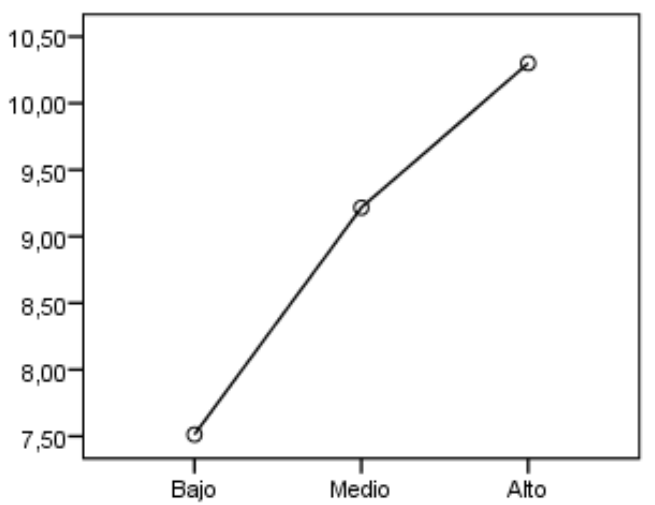

Figura 3. Orientación Extrínseca Personal según niveles de Agresión/Sumisión autoritaria y Convencionalismo. Los valores extremos de la OES son 3 y 15. 
Como puede observarse en la Figura 3, la distribución de la OEP difiere entre la dimensión Agresión/Sumisión y el Convencionalismo, siguiendo el mismo patrón observado en la OI previamente. Con la finalidad de analizar si estas diferencias son estadísticamente significativas se calcularon dos ANOVA de una vía.

Por una parte, la dimensión Agresión/Sumisión diferencia grupos con la OEP $\left(F_{(2,246)}=10.91 ; p<.001\right)$. La misma, a través de un contraste posthoc Tukey $b$, también da cuenta de dos agrupamientos: niveles bajos $(M=7.71)$ y medios $(M=8.70)$ conformaron un grupo, diferenciándose de los que obtuvieron niveles altos ( $M$ $=10.33$ ), quienes conformaron otro grupo. Por otra parte, se observó que la dimensión Convencionalismo diferencia grupos con la OI $\left(F_{(2,246)}=11.48 ; p<\right.$ .001). La OI, a través de un contraste posthoc Tukey $b$, también se divide en dos agrupamientos: puntajes medios $(M=9.41)$ y altos $(M=10.37)$, diferenciándose de quienes obtuvieron niveles bajos $(M=7.51)$.

\section{Discusión}

El análisis de las relaciones entre el autoritarismo y las diferentes expresiones de la religiosidad resulta diferente si se desglosan las dimensiones del autoritarismo. En primer lugar, se observa que las dos dimensiones de RWA se relacionan de forma positiva con la centralidad de la religión, indicando que a mayores niveles de Agresión/ Sumisión autoritaria y Convencionalismo, mayor centralidad de la religión en la vida de los participantes.

Si bien no se han realizado hasta el momento estudios que relacionen las dimensiones del autoritarismo de manera independiente con la centralidad de la religión, debido a que las dos dimensiones estudiadas se relacionan con esta expresión de la religiosidad, los resultados no son diferentes a los informados en estudios previos que evaluaron al autoritarismo con una escala RWA unidimensional (Blogowska \& Saroglou, 2011; Coursey, 2013).

En segundo lugar, al analizar las relaciones entre las dimensiones del autoritarismo con la OI y la OEP, se observaron relaciones positivas y de fuerza media, mientras que con la OES la relación es baja. Los estudios previos que indagaron las relaciones entre estas variables utilizaron la versión original unidimensional de la escala RWA (Altemeyer, 1981). Por ejemplo, Altemeyer y Hunsberger (2005) informan que las OI y OE se asocian con el autoritarismo, aunque la OI lo hace con mayor fuerza. En esa línea, Tsang y Rowatt (2007) hallaron relaciones significativas entre RWA y la OI pero, a diferencia de los resultados informados por Altemeyer y Hunsberger (1992), no observan asociaciones entre el autoritarismo y la OE. Según Maltby (2002), las relaciones entre el autoritarismo y la OE, que caracteriza a quienes utilizan la religión como medio para relacionarse interpersonalmente, dependerían de si la misma es personal o social.

La OEP es una extensión de la orientación intrínseca a las relaciones interpersonales del individuo (Maltby \& Lewis, 1996), por lo tanto, es esperable que se encuentre relacionada con el autoritarismo ya que ofrece un marco privado, normativo y tradicional (Abu-Raiya \& Pargament, 2011). Por el contrario, la OES, al no representar un marco convencional para el individuo (Maltby, 2002), resulta coherente que no presente relaciones con el autoritarismo, tal como ocurre en los resultados informados en el presente trabajo.

$\mathrm{Al}$ dividir los puntajes de las dimensiones del autoritarismo en niveles altos, medios y bajos según sugiere Etchezahar (2012), fue posible observar cómo se distribuyen las orientaciones religiosas según los niveles de cada una de las dos dimensiones de RWA (Agresión/Sumisión autoritaria y Convencionalismo), más allá de las relaciones lineales entre los constructos analizadas previamente (Mavor et al., 2010). De esta manera, fue posible poner de manifiesto que aunque el análisis correlacional indique la presencia de una relación lineal positiva de la OI y la OEP con ambas dimensiones de RWA, al discriminar a estas últimas en niveles (alto, medio y bajo) se observó que las puntuaciones en ambas se comportan de manera diferente en un caso $\mathrm{u}$ otro. Mientras que los niveles medios y altos del Convencionalismo obtienen los mayores puntajes en ambas orientaciones religiosa, en el caso de la Agresión/ Sumisión autoritaria esto sucede sólo con los niveles altos. Por el contrario no se observaron diferencias 
significativas entre las dimensiones de RWA al considerar como factor la Orientación Extrínseca Social, lo cual resulta coherente con el análisis correlacional informado en el apartado anterior.

Estos resultados pueden comprenderse si se tiene en cuenta que más allá de que un individuo pueda vivir su vida sobre la base de un credo religioso o simplemente considerarlo como un marco social de intercambio, ambos aspectos forman parte del Convencionalismo de un individuo. Dicho de otro modo, la religiosidad, no implica a priori una sumisión a un credo religioso determinado, ni la agresión hacia individuos que no lo profesen, ambos componentes centrales del autoritarismo (Altemeyer, 2006).

Los resultados hallados en este estudio realizan un aporte en el análisis de las relaciones entre diferentes expresiones de la religiosidad y el autoritarismo, tanto de forma lineal como no lineal. No obstante, es necesario continuar indagando las relaciones entre los constructos, en particular, considerando las limitaciones de este estudio como la homogeneidad de la muestra con la que se trabajó. Otra de las limitaciones del trabajo es la evaluación de la centralidad de la religión a través de un solo ítem. Si bien hay abundantes estudios que utilizaron esta forma de indagar el concepto (ver Huber \& Huber, 2012), es discutible la validez de constructo que puede ofrecer una evaluación de este tipo. Atendiendo a lo expuesto, futuros trabajos deberían indagar las relaciones entre el autoritarismo y otras evaluaciones de la religiosidad más robustas, así como también analizar si las relaciones entre los constructos varían de acuerdo según diferentes credos religiosos.

\section{Referencias}

Abu-Raiya, H., \& Pargament, K. I. (2011). Putting research into practice: Toward a clinical psychology of religion and spirituality. En I. Noth, C. Morgenthaler, \& K. J. Greider (Eds.), Pastoral psychology and psychology of religion in dialogue (pp. 13-27). Stuttgart, Germany: Verlag W. Kohlhammer.

Allport, G. W., \& Ross, J. M. (1967). Personal religious orientation and prejudice. Journal of Personality and Social Psychology, 5, 432-443.
Altemeyer, B. (1981). Right-Wing Authoritarianism. Winnipeg: University of Manitoba Press.

Altemeyer, B. (1996). The Authoritarian Spectre. Cambridge, MA: Harvard University Press.

Altemeyer, B. (2002). Dogmatic behavior among students: Testing a new measure of dogmatism. Journal of Social Psychology, 142, 713-721.

Altemeyer, B. (2006). The Authoritarians. Winnipeg: University of Manitoba Press.

Altemeyer, B., \& Hunsberger, B. (1992). Authoritarianism, Religious Fundamentalism, Quest, and Prejudice. International Journal for the Psychology of Religion, 2(2), 113-133.

Altemeyer, B., \&Hunsberger,B. (2005). Fundamentalism and authoritarianism. En R. F. Paloutzian \& C. L. Park (Eds.), Handbook of the psychology of religion and spirituality (pp. 378-393). New York, NY: Guildford Press.

Blogowska, J., \& Saroglou, V. (2011). Religious Fundamentalism and Limited Prosociality as a Function of the Target. Journal for the Scientific Study of Religion, 50(1), 44-60.

Coursey, L. (2013). The role of religion in managing existential threat: Effects on outgroup attitudes. Texas: University of Texas at Arlington Press.

Dezutter, J., Robertson, L. A., Luyckx, K., \& Hutsebaut, D. (2010). Life satisfaction in chronic pain patients: The stress-buffering role of the centrality of religion. Journal for the Scientific Study of Religion, 49(3), 507-516.

Etchezahar, E. (2012). Las dimensiones del autoritarismo: Análisis de la escala de autoritarismo del ala de derechas (RWA) en una muestra de estudiantes universitarios de la Ciudad de Buenos Aires. Revista Psicología Política, 12(25), 591-603.

Etchezahar, E., \& Brussino, S. (2013). Psychological perspective on the study of authoritarianism. Journal of Alternative Perspectives in the Social Sciences, 5(3), 495-521. 
Feldman, S. (2003). Enforcing social conformity: A theory of authoritarianism. Political Psychology, 24, $41-74$.

Gorsuch, R. L., \& Venable, G. D. (1983). Development of an "Age universal" IE scale. Journal for the Scientific Study of Religion, 22(2), 181-187.

Huber, S. (2003). Zentralität und Inbalt. Ein neues multidimensionales Messmodell der Religiosität. Opladen: Leske Budrich.

Huber, S., \& Huber, O. (2012). The Centrality of Religiosity Scale (CRS). Religions, 3, 710-724.

Johnson, M. K., Rowatt, W. C., Barnard-Brak, L. M., Patock-Peckham, J. A., LaBouff, J., \& Carlisle, R. D. (2011). Mediational analysis of the role of rightwing authoritarianism and religious fundamentalism in the religion-prejudice link. Personality and Individual Differences, 50, 851-856.

Maltby, J. (2002). The Age Universal I-E Scale-12 and orientation toward religion: Confirmatory factor analysis. The Journal of Psychology, 136(5), 555-560.

Maltby, J., \& Lewis, C. A. (1996). Measuring intrinsic and extrinsic orientation toward religion: Amendments for its use among religious and nonreligious samples. Personality and Individual Differences, 21(6), 937-946.

Marasca, M. M., Marasca, R., \& Imhoff, D. (2013). Indagación del autoritarismo en la infancia: Vinculaciones con la orientación de dominancia social y los valores en niños y niñas de la ciudad de Córdoba (Argentina). Interdisciplinaria, 30(1), 139-161.

Mavor, K. I., Louis, W. R., \& Laythe, B. (2011). Religion, Prejudice, and Authoritarianism: Is RWA a Boon or Bane to the Psychology of Religion? Journal for the Scientific Study of Religion, 50(1), 22-43.
Mavor, K. I., Macleod, C. J., Boal, M. J., \& Louis, W. R. (2009). Right-wing authoritarianism, fundamentalism and prejudice revisited: Removing suppression and statistical artefact. Personality and Individual Differences, 46(5-6), 592-597.

Núñez-Alarcón, M., Moreno-Jiménez, M. P., \& MoralToranzo, F. (2011). Modelo causal del prejuicio religioso. Anales de Psicología, 27, 852-861.

Park, C. L. (2007). Religiousness/Spirituality and health: A meaning systems perspective. Journal of Behavioral Medicine, 30, 319-328.

Sibley, C. G. \& Duckitt,J. (2008). Personality and prejudice: A meta-analysis and theoretical review. Personality and Social Psychology Review, 12, 248-279.

Simkin, H., \& Etchezahar, E. (2013). Las Orientaciones Religiosas Extrínseca e Intrínseca: Validación de la "Age Universal" I-E Scale en el Contexto Argentino. Psykhe, 22(1), 97-106.

Terrizzi, J. A., Shook, N. J., \& Ventis, W. L. (2010). Disgust: A predictor of social conservatism and prejudicial attitudes toward homosexuals. Personality and Individual Differences, 49, 587-592.

Tsang, J., \& Rowatt, W. (2007). The Relationship Between Religious Orientation, Right-Wing Authoritarianism, and Implicit Sexual Prejudice. International Journal for the Psychology of Religion, 17(2), 99-120.

Watson, P. J., Sawyers, P., Morris, R. J., Carpenter, M. L., Jimenez, R. S., Jonas, K. A., \& Robinson, D. L. (2003). Reanalysis within a Christian ideological surround: Relationships of intrinsic religious orientation with fundamentalism and right-wing authoritarianism. Journal of Psychology \& Theology, 31, 315-328.

Recibido: 21 de febrero de 2015

Aceptado: 20 de mayo de 2015 
